\title{
An Improved Self-Starting Implicit Hybrid Method
}

\author{
E. O. Adeyefa \\ Department of Mathematics/Statistics, Federal University Wukari, Taraba State, Nigeria.
}

\begin{abstract}
The paper presents the derivation a hybrid block method for the solutions of Initial Value Problems of Ordinary Differential Equations. This is achieved by using collocation and interpolation technique to contruct a self-starting method with continuous coefficients together with the additional methods from its first derivative which are combined to form a single block that simultaneously provide the approximate solutions for Initial Value Problem. The analysis of the properties of the method such as stability, consistency and convergence are discussed and the performance of the method is demonstrated on two test problems to show the accuracy and efficiency of the method. On comparison of the results obtained from numerical examples with some existing methods, we discovered that the method behaved favourably well.
\end{abstract}

Keywords: Chebyshev polynomial, Collocation, Hybrid, Interpolation.

The class of problems

\section{Introduction}

together with initial conditions

$$
y^{m}(x)=f\left(x, y, y^{\prime}, y^{\prime \prime}, \ldots, y^{m-1}\right)
$$

$$
y^{t}\left(x_{0}\right)=y_{0}^{t}
$$

where $\mathrm{t}=0,1$ and 2 has received much attention by researchers. A lot of research have been carried out to solve higher order ordinary differential equations (ODEs) numerically, but by first reducing it to a system of first order ODEs and then solve by a method designed for a first order ODEs. The deficiencies of this approach, which include time wastage, computational burden and high cost of implementation are extensively discussed in the literature.

Various numerical schemes for solving differential equations exist in literature. Among these are the Runge-kutta, Taylor's algorithm and the Linear Multistep Methods (LMMs). Owing to the suitability of LMMs in providing solutions to ODEs, many scholars have developed LMMs varying from discrete to continuous for the solution of IVPs. However, it has been reported that continuous LMMs has greater advantages over the discrete method in that they give better error estimation and guarantee easy appropriation of solution at all interior points of the integration interval. [1] and [2] considered LMMs where in [2], LMMs were proposed and implemented in a predictor-corrector scheme using the Taylor series algorithm to supply the starting values. Although, the implementation of the methods yielded good accuracy but the procedure is more costly to implement. Many researchers have attempted the solution of this kind of problem using LMMs without reduction to system of first order ODEs, (see [3], [4], [5], [6]). Conventionally, implicit LMMs, when implemented in the predictor-corrector mode is prone to error propagation. This disadvantage has led to the development of block methods from linear multistep methods. Apart from being self-starting, the method does not require the development of the predictors separately, and evaluates fewer functions per step.

Due to the elegant property of Chebyshev polynomial such as equi-oscillation in its entire range of definition and consequent even distribution of error therein we shall employ it to develop a class of finite difference method which is self starting. Its mini-max property also make it desirable.

\section{Material and Methods}

In this section, we set out to derive the proposed continuous hybrid one step block method by approximating the analytical solution of

$$
y^{\prime \prime}=f\left(x, y, y^{\prime}\right), y^{\prime}(a)=z_{0}, y(a)=y_{0}
$$

where $\mathrm{f}$ is a continuous function, with a Chebyshev polynomial in the form

$$
y(x)=\sum_{j=0}^{(r+s)-1} a_{j} T_{j}(x)
$$

on the partition $\mathrm{a}=\mathrm{x}_{0}<\mathrm{x}_{1}<\ldots<\mathrm{x}_{\mathrm{n}}<\mathrm{x}_{\mathrm{n}+1}<\ldots<\mathrm{x}_{\mathrm{N}}=\mathrm{b}$ of the integration interval [a, b], with a constant step size $h$, given by $h=x_{n+1}-x_{n} ; n=0,1, \ldots, N-1$. The second derivative of (2) is given by 


$$
y(x)=\sum_{j=0}^{(r+s)-1} a_{j} T_{j}^{\prime \prime}(x)
$$

where $x \in[a, b]$, the $a_{j}$ 's are real unknown parameters to be determined and $r+s$ is the sum of the number of collocation and interpolation points.

We shall interpolate at at least two points to be able to approximate (2) and for this purpose, we proceed by selecting some offstep points in such a manner that the zero-stability of the main method is guaranteed. Then (2) is interpolated at $\mathrm{x}_{\mathrm{n}+\mathrm{s}}$ and its second derivative is collocated at $\mathrm{x}_{\mathrm{n}+\mathrm{r}}$ so as to obtain a system of equations which will be solved by Gaussian elimination method. The resulting $a_{j}^{\prime} s$ are substituted into (2) to yield the new continuous method after some manipulations. Evaluating the continuous method at the desired point gives the main method. Other methods needed to couple with the main method are to be derived from the continuous method and then solve simultaneously to form the block method.

\subsection{Derivation of Two Offstep Points}

Here, two offstep points (TOP) are introduced. These two points are carefully selected to be $\frac{1}{3}$ and $\frac{2}{3}$ where the collocation point, $r=4$ and the interpolation point, $s=2$.

Applying these in (2), we have

$$
y(x)=\sum_{j=0}^{5} a_{j} T_{j}(x)
$$

with its second derivative given by

$$
y(x)=\sum_{j=0}^{5} a_{j} T_{j}^{\prime \prime}(x)
$$

Substituting (5) into (1) gives

$$
y(x)=\sum_{j=0}^{5} a_{j} T_{j}^{\prime \prime}(x)=f\left(x, y, y^{\prime}\right)
$$

Collocating (6) at $x=x_{n+r}, r=0, \frac{1}{3}, \frac{2}{3}, 1$ and interpolating (4) at $x=x_{n+s}, s=\frac{1}{3}$ and $\frac{2}{3}$ lead to a system of equations written in matrix form $\mathrm{AX}=\mathrm{B}$ as follows:

$$
\left[\begin{array}{cccccc}
1 & \frac{1}{3} & \frac{-7}{9} & \frac{23}{27} & \frac{17}{81} & \frac{-241}{243} \\
1 & \frac{1}{3} & \frac{-7}{9} & \frac{-23}{27} & \frac{17}{81} & \frac{241}{243} \\
0 & 0 & 16 & -96 & 320 & -800 \\
0 & 0 & 16 & -32 & \frac{64}{3} & \frac{3040}{27} \\
0 & 0 & 16 & 32 & \frac{-64}{3} & \frac{-3040}{27} \\
0 & 0 & 16 & 96 & 320 & 800
\end{array}\right]\left[\begin{array}{l}
a_{0} \\
a_{1} \\
a_{2} \\
a_{3} \\
a_{4} \\
a_{5}
\end{array}\right]=\left[\begin{array}{c}
y_{n+\frac{1}{3}} \\
y_{n+\frac{2}{3}} \\
h^{2} f_{n} \\
h^{2} f_{n+\frac{1}{3}} \\
h^{2} f_{n+\frac{2}{3}} \\
h^{2} f_{n+1}
\end{array}\right]
$$

Solving (7) by Gaussian elimination method yields the $a_{j}$ 's as follows: 


$$
\left.\begin{array}{l}
a_{0}=\frac{1}{2} y_{n+\frac{1}{3}}+\frac{1}{2} y_{n+\frac{2}{3}}+\frac{h^{2}}{55296}\left(67 f_{n}+1277 f_{n+\frac{1}{3}}+1277 f_{n+\frac{2}{3}}+67 f_{n+1}\right) \\
a_{1}=\frac{3}{2} y_{n+\frac{2}{3}}-\frac{3}{2} y_{n+\frac{2}{3}}+\frac{h^{2}}{276480}\left(731 f_{n}-8847 f_{n+\frac{1}{3}}+8847 f_{n+\frac{2}{3}}+731 f_{n+1}\right) \\
a_{2}=\frac{h^{2}}{512}\left(f_{n}+15 f_{n+\frac{1}{3}}+15 f_{n+\frac{2}{3}}+f_{n+1}\right) \\
a_{3}=\frac{h^{2}}{12288}\left(135 f_{n+\frac{2}{3}}-135 f_{n+\frac{1}{3}}+19 f_{n}+19 f_{n+1}\right) \\
a_{4}=\frac{3 h^{2}}{2048}\left(f_{n}-f_{n+\frac{1}{3}}-f_{n+\frac{2}{3}}+f_{n+1}\right) \\
a_{5}=\frac{9 h^{2}}{20480}\left(3 f_{n+\frac{1}{3}}-f_{n}-3 f_{n+\frac{2}{3}}+f_{n+1}\right)
\end{array}\right\}
$$

Substituting the $a_{j}$ 's, $j=0$ (Error! Reference source not found.) 5 into (4) yields the continuous hybrid one step method in the form of a continuous linear multistep method described by the formula

$$
y(x)=\alpha_{\frac{1}{3}} y_{n+\frac{1}{3}}+\alpha_{\frac{2}{3}} y_{n+\frac{2}{3}}+h^{2}\left(\beta_{0} f_{n}+\beta_{\frac{1}{3}} f_{n+\frac{1}{3}}+\beta_{\frac{2}{3}} f_{n+\frac{2}{3}}+\beta_{1} f_{n+1}\right)
$$

where $\alpha_{\mathrm{j}}$ 's and $\beta_{\mathrm{j}}$ 's are continuous functions and are obtained as parameters

$$
\left.\begin{array}{l}
\alpha_{\frac{1}{3}}(t)=\frac{1}{2}-\frac{3}{2} t \\
\alpha_{\frac{2}{3}}(t)=\frac{1}{2}+\frac{3}{2} t \\
\beta_{0}(t)=\frac{h^{2}}{34560}\left(25-7 t-270 t^{2}+90 t^{3}+405 t^{4}-243 t^{5}\right. \\
\beta_{\frac{1}{3}}(t)=\frac{-53 h^{2}}{6912}+\frac{29 h^{2}}{3840} t+\frac{9 h^{2}}{128} t^{2}-\frac{9 h^{2}}{128} t^{3}-\frac{3 h^{2}}{256} t^{4}+\frac{27 h^{2}}{1280} t^{5} \\
\beta_{\frac{1}{3}}(t)=\frac{-53 h^{2}}{6912}-\frac{29 h^{2}}{3840} t+\frac{9 h^{2}}{128} t^{2}-\frac{9 h^{2}}{128} t^{3}-\frac{3 h^{2}}{256} t^{4}-\frac{27 h^{2}}{1280} t^{5} \\
\beta_{\frac{1}{3}}(t)=\frac{h^{2}}{34560}\left(25+7 t-270 t^{2}-90 t^{3}+405 t^{4}+243 t^{5}\right.
\end{array}\right\}
$$

where $\mathrm{t}=\frac{2 v-h}{h}$ and $\mathrm{v}=\mathrm{x}-\mathrm{x}_{\mathrm{n}}$

Evaluating (9) at $\mathrm{x}=\mathrm{x}_{\mathrm{n}}$ and $\mathrm{x}_{\mathrm{n}+1}$, we obtain the discrete methods from (10) as follows:

$$
y_{n+1}=2 y_{n+\frac{2}{3}}-y_{n+\frac{1}{3}}+\frac{h^{2}}{108}\left(f_{n+1}+10 f_{n+\frac{2}{3}}+f_{n+\frac{1}{3}}\right)
$$

and

$$
y_{n}=2 y_{n+\frac{1}{3}}-y_{n+\frac{2}{3}}+\frac{h^{2}}{108}\left(f_{n}+10 f_{n+\frac{1}{3}}+f_{n+\frac{2}{3}}\right)
$$

The block methods are derived by evaluating the first derivative of (9) in order to obtain additional equations needed to couple with (11) and (12).

Differentiating (9), we obtain 
$y^{\prime}(x)=\frac{1}{h}\left(\alpha_{\frac{1}{3}}^{\prime} y_{n+\frac{1}{3}}+\alpha_{\frac{2}{3}}^{\prime} y_{n+\frac{2}{3}}\right)+h\left(\beta_{0}^{\prime} f_{n}+\beta_{\frac{1}{3}}^{\prime} f_{n+\frac{1}{3}}+\beta^{\prime}{ }_{\frac{2}{3}} f_{n+\frac{2}{3}}+\beta_{1}^{\prime} f_{n+1}\right)$

where

$$
\left.\begin{array}{l}
\alpha_{\frac{1}{3}}^{\prime}(t)=\frac{-3}{h} \\
\alpha_{\frac{2}{3}}^{\prime}(t)=\frac{3}{h} \\
\beta_{0}{ }_{\frac{1}{3}}(t)=\frac{-7 h}{17280}-\frac{h}{32} t+\frac{h}{64} t^{2}+\frac{3 h}{32} t^{2}-\frac{9 h}{128} t^{4} \\
\beta_{\frac{1}{3}}^{\prime}(t)=\frac{29 h}{1920}+\frac{9 h}{32} t-\frac{27 h}{64} t^{2}-\frac{3 h}{32} t^{3}+\frac{27 h}{128} t^{4} \\
\beta_{\frac{2}{3}}^{\prime}(t)=\frac{-29 h}{1920}+\frac{9 h}{32} t+\frac{27 h}{64} t^{2}-\frac{3 h}{32} t^{2}-\frac{27 h}{128} t^{4} \\
\beta_{1}^{\prime}(t)=\frac{7 h}{17280}-\frac{h}{32} t-\frac{h}{64} t^{2}+\frac{3 h}{32} t^{3}+\frac{9 h}{128} t^{4}
\end{array}\right\}
$$

Evaluating (14) at $\mathrm{x}=\mathrm{x}_{\mathrm{n}}, \mathrm{x}_{\mathrm{n}+1}, \mathrm{x}_{\mathrm{n}+2}$ and $\mathrm{x}_{\mathrm{n}+1}$, the following discrete derivative schemes are obtained.

$$
\left.\begin{array}{l}
1080 h y_{n}^{\prime}-3240 y_{n+\frac{2}{3}}+3240 y_{n+\frac{1}{3}}=h^{2}\left(-127 f_{n}-414 f_{n+\frac{1}{3}}+9 f_{n+\frac{2}{3}}-8 f_{n+1}\right) \\
1080 h y_{n+\frac{1}{3}}^{\prime}-3240 y_{n+\frac{2}{3}}+3240 y_{n+\frac{1}{3}}=h^{2}\left(8 f_{n}-129 f_{n+\frac{1}{3}}-66 f_{n+\frac{2}{3}}+7 f_{n+1}\right) \\
1080 h y_{n+\frac{2}{3}}^{\prime}-3240 y_{n+\frac{2}{3}}+3240 y_{n+\frac{1}{3}}=h^{2}\left(-7 f_{n}+66 f_{n+\frac{1}{3}}+129 f_{n+\frac{2}{3}}-8 f_{n+1}\right) \\
1080 h y_{n+1}^{\prime}-3240 y_{n+\frac{2}{3}}+3240 y_{n+\frac{1}{3}}=h^{2}\left(8 f_{n}-9 f_{n+\frac{1}{3}}+414 f_{n+\frac{2}{3}}+127 f_{n+1}\right)
\end{array}\right\}
$$

Equations (11), (12) and (15) are combined and solved simultaneously to obtain the following explicit results.

$$
\left.\begin{array}{l}
y_{n+\frac{1}{3}}=y_{n}+\frac{1}{3} h y_{n}^{\prime}+\frac{h^{2}}{3240}\left(8 f_{n+1}-39 f_{n+\frac{2}{3}}+114 f_{n+\frac{1}{3}}+97 f_{n}\right) \\
y_{n+\frac{2}{3}}=y_{n}+\frac{2}{3} h y_{n}^{\prime}+\frac{h^{2}}{405}\left(2 f_{n+1}-6 f_{n+\frac{2}{3}}+66 f_{n+\frac{1}{3}}+28 f_{n}\right) \\
y_{n+1}=y_{n}+h y_{n}^{\prime}+\frac{h^{2}}{120}\left(2 f_{n+1}+9 f_{n+\frac{2}{3}}+36 f_{n+\frac{1}{3}}+13 f_{n}\right) \\
y_{n+\frac{1}{3}}^{\prime}=y_{n}^{\prime}+\frac{h}{72}\left(f_{n+1}-5 f_{n+\frac{2}{3}}+19 f_{n+\frac{1}{3}}+9 f_{n}\right) \\
y_{n+\frac{2}{3}}^{\prime}=y_{n}^{\prime}+\frac{h}{9}\left(f_{n+\frac{2}{3}}+4 f_{n+\frac{1}{3}}+f_{n}\right) \\
y_{n+1}^{\prime}=y_{n}^{\prime}+\frac{h}{8}\left(f_{n+1}+3 f_{n+\frac{2}{3}}+3 f_{n+\frac{1}{3}}+f_{n}\right)
\end{array}\right\}
$$

Equation (16) recovers the dicrete counterpart when power series was used as basis function in [8]. 


\section{Numerical Examples}

We consider here two test problems for the efficiency and accuracy of the method implemented as a block method. The absolute errors of the test problems are compared with our earlier work.

\subsection{Problems}

Problem 1

$$
\begin{aligned}
& y^{\prime \prime}=y^{\prime}, y(0)=0, y^{\prime}(0)=-1 \\
& \text { Exact Solution: } y(x)=1-e^{x}
\end{aligned}
$$

Source:Yahaya and Badmus (2009).

Probem 2

$$
\begin{aligned}
& y^{\prime \prime}=2 y-y^{\prime}, y(0)=0, y^{\prime}(0)=1 \\
& \text { Exact Solution: } y(x)=\frac{e^{x}-e^{-2 x}}{3} \\
& \text { Source: Adeniyi et al (2008). }
\end{aligned}
$$

\section{Results}

TABLE 1a: Showing the exact solutions and the computed results for problem 1

\begin{tabular}{|c|c|c|}
\hline $\mathrm{X}$ & Exact Solution & Two Offstep Points \\
\hline 0.1 & -0.105170918 & -0.105170918 \\
\hline 0.2 & -0.221402758 & -0.221402759 \\
\hline 0.3 & -0.349858807 & -0.349858808 \\
\hline 0.4 & -0.491824697 & -0.491824699 \\
\hline 0.5 & -0.64872127 & -0.64872127 \\
\hline 0.6 & -0.8221188 & -0.822118804 \\
\hline 0.7 & -1.013752707 & -1.013752713 \\
\hline 0.8 & -1.225540928 & -1.225540937 \\
\hline 0.9 & -1.459603111 & -1.459603122 \\
\hline 1.0 & -1.718281828 & -1.718281844 \\
\hline
\end{tabular}

TABLE 1b: Comparison of absolute errors for Problem 1

\begin{tabular}{|l|l|l|}
\hline $\mathrm{X}$ & Error in [7], $\mathrm{p}=4, \mathrm{k}=1$ & Error in TOP, $\mathrm{p}=4, \mathrm{k}=1$ \\
\hline 0.1 & $0.160756 \mathrm{E}-07$ & $0.847212855 \mathrm{e}-012$ \\
\hline 0.2 & $0.351602 \mathrm{E}-07$ & $0.35792513310 \mathrm{e}-011$ \\
\hline 0.3 & $0.237576 \mathrm{E}-06$ & $0.8583191935-011$ \\
\hline 0.4 & $0.2646413 \mathrm{E}-06$ & $0.1630669488 \mathrm{e}-010$ \\
\hline 0.5 & $0.2967001 \mathrm{E}-06$ & $0.2726645687 \mathrm{e}-010$ \\
\hline 0.6 & $0.3343905 \mathrm{E}-06$ & $0.4205776616 \mathrm{e}-010$ \\
\hline 0.7 & $0.3784705 \mathrm{E}-06$ & $0.6136532837 \mathrm{e}-010$ \\
\hline 0.8 & $0.4304925 \mathrm{E}-06$ & $0.8597550005 \mathrm{e}-010$ \\
\hline 0.9 & $0.4911569 \mathrm{E}-06$ & $0.1167900776 \mathrm{e}-009$ \\
\hline 1.0 & $0.561459 \mathrm{E}-06$ & $0.1548418993 \mathrm{e}-009$ \\
\hline
\end{tabular}

TABLE 2a: Showing the exact solutions and the computed results for problem 2

\begin{tabular}{|l|l|l|}
\hline $\mathrm{X}$ & Offstep2 & Exact \\
\hline 0.1 & 0.095480053582243 & 0.095480054999222 \\
\hline 0.2 & 0.183694232119942 & 0.183694237374844 \\
\hline 0.3 & 0.267015712944721 & 0.267015723827326 \\
\hline 0.4 & 0.347498559993963 & 0.347498577841350 \\
\hline
\end{tabular}

TABLE 2b: Comparison of absolute errors for Problem 2

\begin{tabular}{|l|l|l|}
\hline $\mathrm{X}$ & Error in TOP & Error in [9] \\
\hline 0.1 & $1.416978520629719 \mathrm{e}-009$ & $3.7135900 \mathrm{e}-4$ \\
\hline 0.2 & $5.254901042084370 \mathrm{e}-009$ & $1.5836410 \mathrm{e}-3$ \\
\hline 0.3 & $1.088260487280834 \mathrm{e}-008$ & $3.4173760 \mathrm{e}-3$ \\
\hline 0.4 & $1.784738623555882 \mathrm{e}-008$ & $5.7170630 \mathrm{e}-3$ \\
\hline
\end{tabular}




\section{Conclusion}

This paper has demonstrated the derivation of continuous two-offstep hybrid method for the direct integration of second order ordinary differential equations. It has been observed through comparison of the solutions of the selected test problems with solutions obtained in our earlier paper, [10] that increase in the number of offstep points leads to increase in the efficiency and accuracy of the method. Moreover, the desirable property of a numerical solution is to behave like the theoretical solution of the problem as this is vivid in the Tables shown above. In the future paper, the scope of the paper shall be extended to hybrid two step points.

\section{References}

[1] Vigo-Aguiar, J. and Ramos, H., Dissipative Chebyshev exponential-fitted methods for numerical solution of second-order differential equations, J. Comput. Appl. Math. 158, 2003, 187-211.

[2] Awoyemi, D.O., A class of Continuous Methods for general second order initial value problems in ordinary differential equation. International Journal of Computational Mathematics, 72, 1999, 29-37.

[3] Bun, R.A. and Varsil'yer, Y.D., A numerical method for solving differential equations of any orders. Comp. Math. Phys. 32(3), 1992, 317 - 330.

[4] Lambert, J.D., Numerical Methods for Ordinary Differential Systems. John Wiley, New York, 1991.

[5] Kayode S.J., An improve numerov method for direct solution of general second order initial value problems of ODEs, National Mathematical Centre proceedings 2005.

[6] Adesanya, A.O., Anake, T.A., Bishop, S.A. and Osilagun, J.A., Two Steps Block Method for the solution of general second order Initial Value Problems of Ordinary Differential Equations. Journal of Natural Sciences, Engineering and Technology, J. Nat. Sci. Engr. Tech., 8(1), 2009, 25 - 33.

[7] Yahaya, Y. A. and Badmus, A. M., A Class of Collocation Methods for General Second Order Ordinary Differential Equations. African Journal of Mathematics and Computer Science research, 2(4), 2009, 069 - 072.

[8] Anake T.A., Continuous Implicit Hybrid One-Step Methods for the solutions of Initial Value Problems of general second order Ordinary Differential Equations. Ph.D Thesis (Unpublished) Covenant University, Ota, Nigeria, 2011.

[9] Adeniyi, R.B., Alabi, M.O. and Folaranmi, R.O., A Chebyshev collocation approach for a continuous formulation of hybrid methods for initial value problems in ordinary differential equations. Journal of the Nigerian Association of Mathematical Physics, $12,2008,369-378$

[10] Adeyefa E.O. and Adeniyi R.B., Chebyshev Collocation Approach for a Continuous Formulation of Implicit Hybrid Methods for IVPs in Second Order ODEs. International Organisation of Scientific Research Journal of Mathematics, 6(4), 2013, 09-12. 